\title{
Controlled polymer dewetting by physical confinement
}

\author{
K. Y. Suh, Joonhyung Park, and Hong H. Lee $\mathrm{e}^{\mathrm{a})}$ \\ School of Chemical Engineering, Seoul National University, Seoul, 151-742, Korea
}

(Received 9 October 2001; accepted 14 February 2002)

\begin{abstract}
We report ordering of polymer drops that takes place when a thin polystyrene film confined by polydimethylsiloxane walls dewets on a silicon substrate. When annealed above the glass transition temperature, the thin polymer film dewets, resulting in the formation of a regular structure inside and outside the confinement. It is found that the ordering becomes strongly suppressed as the film thickness increases and the pattern size decreases due to the physical confinement of the mold. As a result, the ordering can take place only when the wavelength of the capillary wave is smaller than the characteristic length of the physical confinement. (C) 2002 American Institute of Physics.
\end{abstract}

[DOI: $10.1063 / 1.1467898$ ]

\section{INTRODUCTION}

Thermal stability and structure formation of thin films have extensively been studied for their technological importance in applications as in coatings, adhesives, and dielectric layers. ${ }^{1-8}$ Moreover, it can provide a useful pathway to fabricating nanostructures that are difficult to realize by the photolithographic method. ${ }^{9,10}$

Much effort has been devoted to the dewetting behavior of thin polymer films in two-dimensional systems. ${ }^{2,3}$ The polymer films in such systems undergo dewetting above the glass transition temperature $\left(T_{g}\right)$ through the generation of spherical drops with a finite contact angle given by Young's equation. The drops, in turn, contact one another, resulting in the well-known cellular structure. ${ }^{2}$ The usefulness of the structure is restricted in that it lacks long-range order even on a several-micron scale.

Much work in the direction of getting order into dewetting phenomenon has been carried out. ${ }^{11-15}$ Meyer and Braun $^{11}$ generated ordered polymer drops on chemically modified surface by microcontact printing. It was reported that the holes are formed exclusively on the hydrophilic microprinted areas due to poor wettability with the polymer film. Kargupta and Sharma ${ }^{12}$ subsequently developed a theoretical explanation for the controlled dewetting process on a chemically heterogeneous striped pattern. They found that the film breakup is suppressed on some potentially destabilizing nonwettable sites when the pattern spacing is below a certain characteristic length. However, less is known about the dewetting dynamics when the polymer film is physically confined. In this paper, we report on polymer dewetting for physically confined systems.

Micro- or nanostructures of a polymer can be fabricated by placing a polydimethylsiloxane (PDMS) mold with channel patterns on a spin-coated polymer surface and then raising the temperature above $T_{g}$, as shown in Fig. $1 .{ }^{16}$ During the annealing process, the polymer flows into the channel of the mold and forms the replica after mold removal. If one

\footnotetext{
a) Author to whom correspondence should be addressed. Electronic mail: honghlee@plaza.snu.ac.kr
}

hopes to obtain the exact replica of the mold, a high molecular weight polymer should be used as the dewetting of the polymer is quite suppressed throughout the annealing process due to its high viscosity. On the other hand, if one hopes to utilize the dewetting phenomenon of the polymer film, a low molecular weight polymer should be used for the purpose. This latter case is investigated.

\section{EXPERIMENTAL METHOD}

We fabricated PDMS (Sylgard 184, Dow Corning) mold that has a planar surface with protruding (positive) or recessed (negative) patterns by casting PDMS against a complementary relief structure prepared by the photolithographic method. ${ }^{17}$ For the polymer, we used a standard polystyrene (PS) $\left(M_{w}=3900, M_{w} / M_{n}<1.02\right.$, and $\left.T_{g}=98^{\circ} \mathrm{C}\right)$. The low molecular weight used in our experiment facilitates the dewetting process due to its low viscosity. A silicon wafer was cleaned by ultrasonic treatment in trichloroethylene and methanol for 5 min each and dried in nitrogen. Native oxide was not removed of the surface and thus would exist on the surface. Thin films were prepared by spin coating a toluene solution of the polymer onto a silicon substrate. The film thickness ranges from 23 to $75 \mathrm{~nm}$, which was determined by elipsometry with an accuracy of $0.5 \sim 1 \mathrm{~nm}$. The mold with the pattern is placed on the surface of a polymer layer that has been spin coated onto a substrate and then heated above the glass transition temperature (typically $150{ }^{\circ} \mathrm{C}$ ) of the polymer. In the dewetting, the PDMS walls act as a boundary, thereby generating a three-dimensionally confined system. A schematic diagram of our experimental setup is shown in Fig. 1.

\section{RESULTS AND DISCUSSION}

Figure 2 shows various final morphologies of the polymer drops resulting from the dewetting process. A PDMS mold with a recessed box-and-cross pattern (negative mold) was used for the result in Fig. 2(a) and molds with a protruding box and with a cylindrical pattern (positive molds) were used, respectively, for the results in Figs. 2(b) and 2(c). The film thickness is $23 \mathrm{~nm}$ throughout the figures. During the 

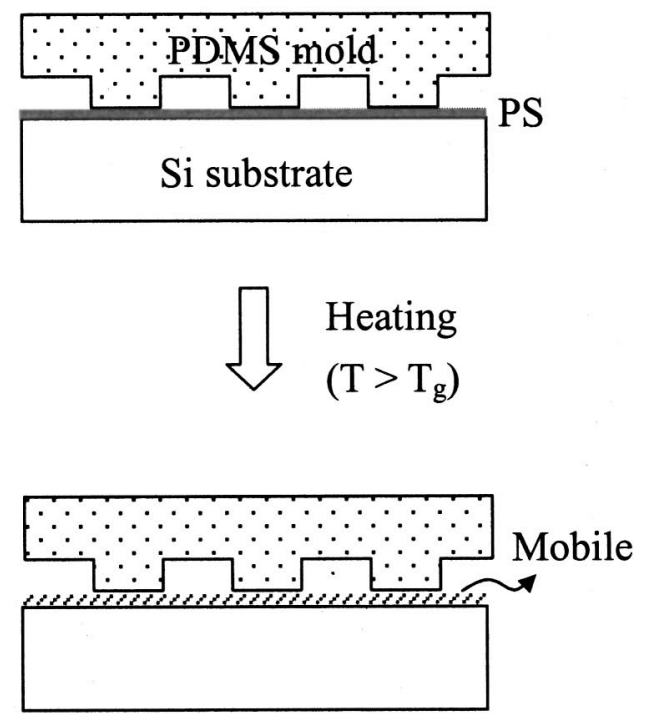

$\lceil$ Mold removal

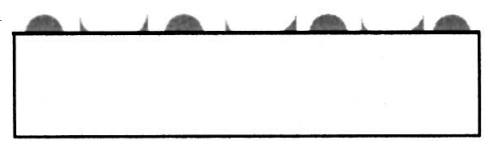

FIG. 1. An illustration of our experimental procedure. For convenience, the PDMS mold is depicted as one with a protruding pattern.

annealing process, most of the dewetted polymer contributes to the formation of polymer drops but a fraction of it climbs up the confining walls due to capillary force. In other words, the PS outside and inside the pattern simultaneously participates in the capillary rise. However, the fraction of the mass from under the mold that is in contact with the polymer is considerably smaller than that from outside the mold due to the hindrance of mass movement by the mold confinement. The thin lines defining the boxes and crosses visible in Fig. 2(a) and rather thick lines defining the boxes in Fig. 2(b) are due to the polymer risen by the capillary force. ${ }^{16}$ The height of the risen polymer ranges from 120 to $350 \mathrm{~nm}$ depending on the pattern size and depth. In the case of the negative mold, the polymer agglomerates to the center of the confinement in order to minimize the interaction energy between the mold and the polymer if the pattern size is sufficiently large as in Fig. 2(a) (a $50 \mu \mathrm{m} \times 50 \mu \mathrm{m}$ box). For the positive molds as in Figs. 2(b) (a $4 \mu \mathrm{m} \times 4 \mu \mathrm{m}$ box) and 2(c) $(2 \mu \mathrm{m}$ diam cylinder), the polymer that is not in contact with the mold, which is the polymer outside the boxes, dewets and grows to large drops that are located between the boxes. The location is determined by the minimum of the interaction energy. Frequently, the drops do not form at the exact middle points between the boxes due to imperfect adhesion conditions. In the figure, the drops are located near one side of a row of boxes or some of the drops form two rows [the upper left corner of Fig. 2(b)]. Outside the molded regions, we observe the conventional cellular structure. ${ }^{2}$
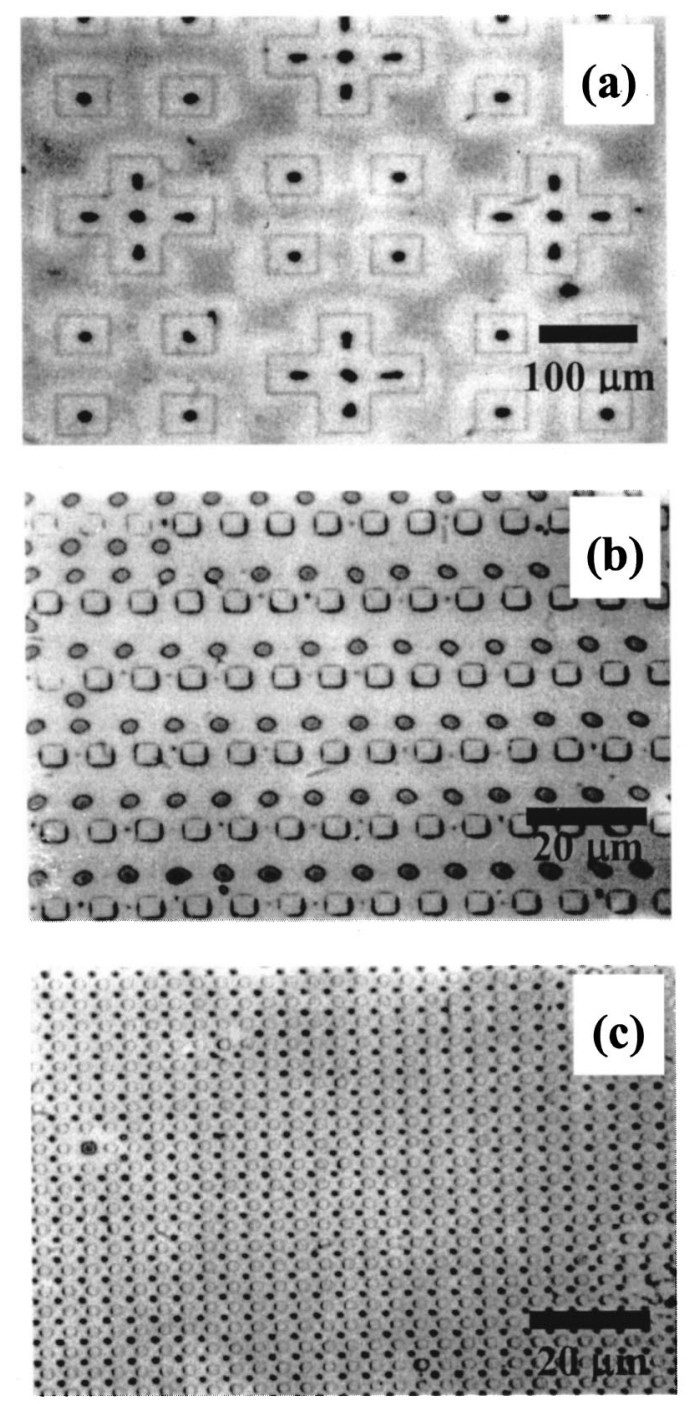

FIG. 2. Optical micrographs of final morphologies of ordered polystyrene drops for (a) recessed (negative) $50 \mu \mathrm{m} \times 50 \mu \mathrm{m}$ box and $150 \mu \mathrm{m}$ $\times 150 \mu \mathrm{m}$ cross patterns, (b) protruding (positive) $4 \mu \mathrm{m} \times 4 \mu \mathrm{m}$ box pattern, and (c) protruding (positive) $2 \mu \mathrm{m}$ diameter cylinder pattern.

To investigate the transients of the regular drop formation, we carried out dewetting experiments with $23 \mathrm{~nm}$ PS films using a positive mold for various annealing times (20, $40,60$, and $80 \mathrm{~min})$ at $130^{\circ} \mathrm{C}$, the results of which are shown in Fig. 3. Both the box size and the space between two adjacent boxes in the figure are $10 \mu \mathrm{m}$. After $20 \mathrm{~min}$ [Fig. 3(a)], the mass climbs up the walls due to capillarity, leading to the thin square lines defining the boxes. Hole formation, which is frequently observed in the conventional dewetting experiments, ${ }^{2,3}$ was not detected in this initial stage. Instead, the polymer recedes from the confining walls both inside and outside the box such that outside the box a large circular rim surrounding the box is observed but a flat and circular drop forms inside the box. Note in this regard that the box in contact with the polymer acts as a defectlike site from which the mass is driven away. The circular rim outside the box is formed by the mass accumulation from the walls, which has the same origin as in the hill formation around the holes in the conventional dewetting. In this initial stage, no distinct 

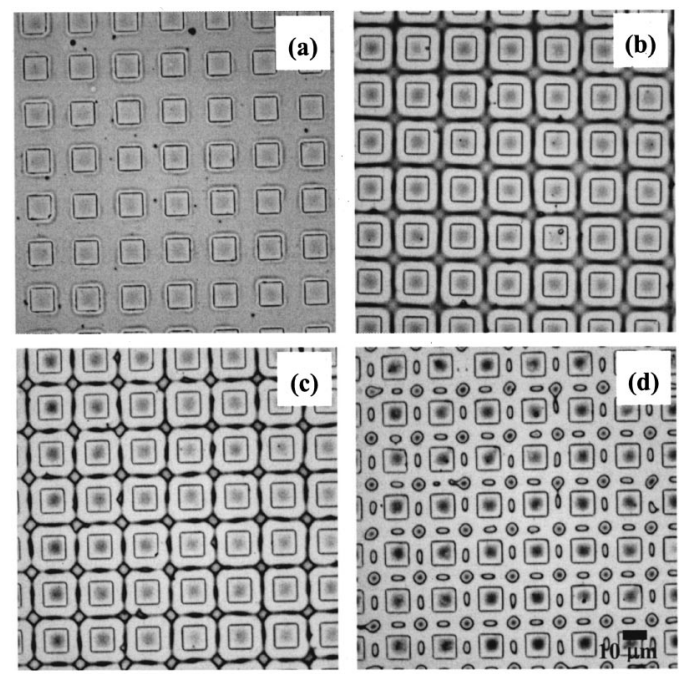

FIG. 3. Optical micrographs of the transient morphologies of polymer drops for a $10 \mu \mathrm{m} \times 10 \mu \mathrm{m}$ positive box pattern. Samples were annealed at $130{ }^{\circ} \mathrm{C}$ for (a) 20, (b) 40, (c) 60, and (d) $80 \mathrm{~min}$, respectively.

ordering is observed. Then, the polymer merges into the thick, square ribbon outside the box. Spherical drops form at four corners due to mass accumulation [Fig. 3(b)]. The drops continuously grow in size at the expense of the ribbons and, finally, the ribbons break up after about $60 \mathrm{~min}$ [Fig. 3(c)]. When the annealing time exceeded $60 \mathrm{~min}$, the drops further merged into larger ones that are located at four corners and four centerlines around a box [Fig. 3(d)], which resembles the two-dimensional projection of a face-centered cubic (FCC) structure. If we assume that the drop shape is a truncated sphere, the contact angle can be calculated from the cross sectional profile of atomic force microscopy (AFM) image to give a value of about $27^{\circ}$, which is in satisfactory agreement with the reported value $\left(20^{\circ}-40^{\circ}\right){ }^{2}$ No further transient morphology was observed even after 1 day such that the structure in Fig. 3(d) appears to be an equilibrium one.

One notable finding in Fig. 3(d) is that the shape of the drops located at the midpoints between corners is oval rather than circular whereas the drops at four corners are circular. This fact reveals that kinetic rather than thermodynamic factors could govern the drop shape. Although a perfectly spherical drop may be thermodynamically stable, the drop cannot change its overall shape once the oval shape forms, possibly because the polymer would migrate an excessive amount to change the overall shape, which might be kinetically hindered because of its high kinetic barrier. Further study would be required to elucidate the effect of physical confinement on the drop shape.

We found in our experiment that polymer drops cease to form as the film thickness increases and the pattern size decreases. A typical example is shown in Fig. 4 for the thickness dependence of the drop formation. The film thicknesses are $23 \mathrm{~nm}$ for Fig. 4(a) and $55 \mathrm{~nm}$ for Fig. 4(b), respectively. A careful examination of Fig. 4 reveals that the amount of mass risen by the capillarity is nearly the same for both cases, which indicates that the thickness of the residual film plays an important role for the ordering. As seen in the fig-
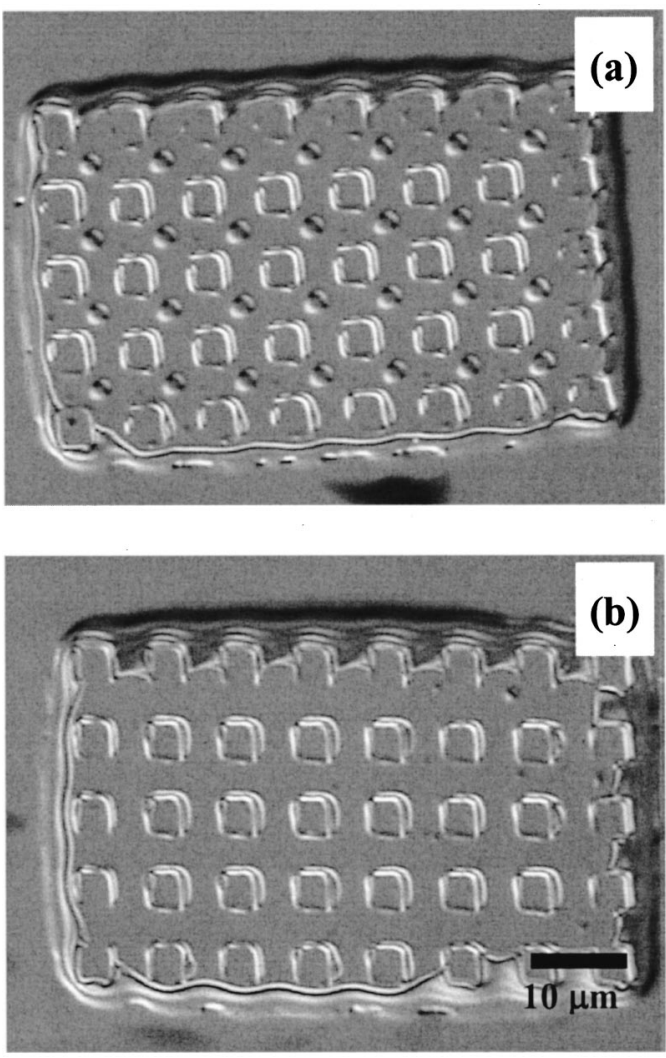

FIG. 4. An example of the effect of film thickness on the drop formation. No drop formation occurs when the film is relatively thick. Film thicknesses are $23 \mathrm{~nm}$ for (a) and $55 \mathrm{~nm}$ for (b), respectively.

ure, the drop formation is totally suppressed for the thicker film $(55 \mathrm{~nm})$.

To investigate the effect of the film thickness and the pattern size on the ordering behavior, we consider propagation of the wave as determined by the balance between surface energy and intermolecular forces. In our experiment, the mold walls act as a physical barrier. Therefore, a wave could not be sustained due to the barrier if the wavelength is larger than the pattern size. This supposition is similar to the conclusion reached by Kargupta and Sharma. ${ }^{12}$ Through simulation of dewetting on chemically heterogeneous stripes, they concluded that the film breakup is suppressed when the pattern spacing is below a certain critical length. The wavelength of the capillary wave can be determined from the capillary wave theory, ${ }^{1}$ the cutoff wave vector $q_{c}$ being given by

$$
q_{c}=\left(\frac{A_{\mathrm{eff}}}{2 \pi \gamma h_{r}{ }^{4}}\right)^{1 / 2},
$$

where $A_{\text {eff }}$ is the effective Hamaker constant for the van der Waals interaction of the film with the surrounding media, $\gamma$ is the surface tension of the film, and $h_{r}$ is the thickness of the film remaining after the capillary rise. In the capillary wave theory, the intermolecular forces acting across the airfilm-substrate system facilitate the local fluctuation of the film thickness $\left(A_{\text {eff }}>0\right)$ to lower the overall free energy. On the other hand, this thickness fluctuation costs additional surface energy such that the surface tension suppresses the dew- 


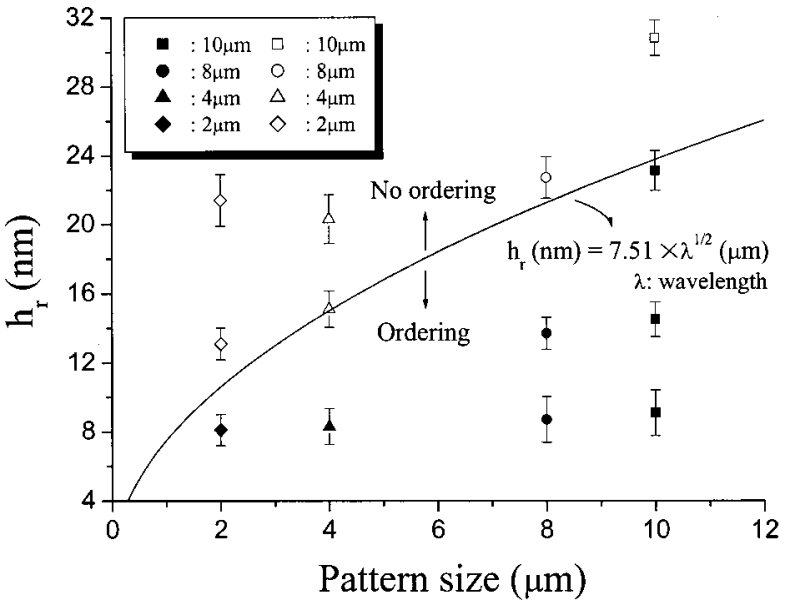

FIG. 5. A comparison of the calculated wavelength of the capillary wave with the pattern size as a function of the thickness of residual film. In the figure, filled symbols indicate ordering and open symbols no ordering. Note that the ordering occurs only when the wavelength is smaller than the pattern size.

etting. Therefore, the cutoff wave vector is determined by the trade-off between the intermolecular forces and the surface tension.

The supposition here is that no polymer drops form when the wavelength, as determined by the cutoff wave vector, is larger than the pattern size, which in the experiment is the pattern width. Otherwise, ordered drops do form. To test the supposition, we carried out experiments in which the pattern size and the film thickness were varied. Shown in Fig. 5 is the comparison between theory and experiment. Given the pattern size, the residual film thickness is a function of the initial film thickness, which ranges from 23 to 75 $\mathrm{nm}$ in our experiment. For the $2 \mu \mathrm{m}$ pattern in Fig. 5, for instance, the initial film thickness was varied for three different residual film thicknesses. The demarcation line in the figure is given by the capillary wave theory [Eq. (1)]. As shown in the figure, $h_{r}(\mathrm{~nm})=7.51 \times \lambda^{1 / 2}(\mu \mathrm{m})$ such that the residual film thickness is equivalent to the wavelength $\lambda$. When the pattern size is smaller than the wavelength (upper region), the capillary wave is blocked, resulting in no drop formation and thus no ordering. On the other hand, ordering can be observed when the pattern size is larger than the wavelength (lower region). It is seen that the theoretical prediction is in satisfactory agreement with the experimental data.

A few comments are in order here. The effective Hamaker constant for the $\mathrm{PS} / \mathrm{SiO}$ system is given by the mixing rule involving individual Hamaker constants as follows:

$$
A_{\mathrm{eff}}=\left(\sqrt{A_{\mathrm{SiO}}}-\sqrt{A_{\mathrm{PDMS}}}\right)\left(\sqrt{A_{\mathrm{air}}}-\sqrt{A_{\mathrm{PDMS}}}\right) .
$$

From the literature, $A_{\mathrm{SiO}}=2.2 \times 10^{-20}(\mathrm{~J}),{ }^{18} A_{\mathrm{PDMS}}=5.2$ $\times 10^{-20}(\mathrm{~J}),{ }^{19}$ and $A_{\text {air }}=0$, such that $A_{\text {eff }}=2.37 \times 10^{-20}(\mathrm{~J})$. The surface tension of the low molecular weight PS (MW $=3900$ ) is about $30 \mathrm{~mJ} / \mathrm{m}^{2}{ }^{20}$ The film thickness remaining after the capillary rise or the residual film thickness, $h_{r}$ in Eq. (1), was determined from an analysis of AFM images. One might think that the residual film thickness could be calculated by counterbalancing the driving force for the capillary rise along the mold, which results in the thinning of the film, against the intermolecular forces between the polymer and the substrate. There are problems in taking this approach. In the first place, a small fraction of the PS under the mold is squeezed to rise along the mold by the capillarity. Therefore, it is difficult to predict the exact portion of the mass from outside the box. The second reason is that the polymer on the PDMS walls is frequently thermally unstable such that it merges into one large drop. ${ }^{19}$ In addition to these complexities, the mass transported by the capillary rise also depends strongly on extrinsic factors such as the wetting property of the mold and substrate conditions. In spite of these problems, the residual film thickness can be calculated from the volume of each polymer drop around and near the box from an analysis of AFM images and the initial film thickness. Note in this regard that the dewetting starts taking place only after the capillarity ceases to play a role, as shown by the transient morphology in Fig. 3.

\section{IN SUMMARY}

It has been shown that PS films of low molecular weight form ordered drops when annealed above the glass transition temperature under the physical confinement of PDMS mold. The shape of the drop located at the midpoints between the corners of boxes is determined by kinetic factors. As a result, an oval rather than the expected circular shape is observed, which opens up a new possibility of controlling the drop shape. It is found that the dewetting is observed only when the wavelength of the capillary wave for the residual film is smaller than the pattern size, which indicates that the mold walls block the propagation of the capillary wave. A theoretical model has been found to adequately describe the ordering. Since the findings should be equally applicable to a number of polymers, the results reported here could provide a convenient way of studying dewetting behavior of thin polymer films under confined geometry and also of fabricating ordered polymer microstructures.

${ }^{1}$ F. Brochard-Wyart and J. Daillant, Can. J. Phys. 68, 1084 (1990).

${ }^{2}$ G. Reiter, Phys. Rev. Lett. 68, 75 (1992).

${ }^{3}$ R. Xie, A. Karim, J. F. Douglas, C. C. Han, and R. A. Weiss, Phys. Rev. Lett. 81, 1251 (1998).

${ }^{4}$ H. Gau, S. Herminghaus, P. Lenz, and R. Lipowsky, Science 283, 46 (1999).

${ }^{5}$ J. J. Lisari, Plastic Coatings for Electronics (McGraw-Hill, New York, 1970).

${ }^{6}$ J. M. G. Cowie, Polymer: Chemistry and Physics of Modern Materials (Chapman and Hall, New York, 1970).

${ }^{7}$ H. Liu, A. Bhattacharya, and A. Chakrabarti, J. Chem. Phys. 109, 8607 (1998)

${ }^{8}$ K. Y. Suh and H. H. Lee, J. Chem. Phys. 115, 8204 (2001).

${ }^{9}$ A. M. Higgins and R. A. L. Jones, Nature (London) 404, 476 (2000).

${ }^{10}$ X. Hu, D. G. Cahill, and R. S. Averback, Appl. Phys. Lett. 76, 3215 (2000).

${ }^{11}$ E. Meyer and H.-G. Braun, Macromol. Mater. Eng. 276/277, 44 (2000).

${ }^{12}$ K. Kargupta and A. Sharma, Phys. Rev. Lett. 86, 4536 (2001).

${ }^{13}$ O. Karthaus, L. Grasjö, N. Maruyama, and M. Shimomura, Chaos 9, 308 (1999).

${ }^{14}$ E. Schäffer, T. Thurn-Albrecht, T. P. Russell, and U. Steiner, Nature (London) 403, 874 (2000).

${ }^{15}$ S. Y. Chou, L. Zhuang, and L. Guo, Appl. Phys. Lett. 75, 1004 (1999).

${ }^{16}$ K. Y. Suh, Y. S. Kim, and H. H. Lee, Adv. Mater. 13, 1386 (2001). 
${ }^{17}$ Y. Xia, J. A. Rogers, K. E. Paul, and G. M. Whitesides, Chem. Rev. 99, 1823 (1999).

${ }^{18}$ J. Isaellachivili, Intramolecular Surfaces Forces, 2nd ed. (Academic, New York, 1992).
${ }^{19}$ M. O. David, G. Reiter, T. Sitthaï, and J. Schultz, Langmuir 14, 5667 (1998).

${ }^{20}$ J. Brandup and E. H. Immergut, Polymer Handbook (Wiley, New York, 1989). 